\title{
Reanclaje medular en pacientes con mielomeningocele y lipomeningocele: la segunda operación
}

\author{
J.F. Martínez-Lage*,**; A. Ruiz-Espejo Vilar*; M.J. Almagro*; I. Sánchez del Rincón***; J. Ros de San Pedro*; M. \\ Felipe-Murcia*y F.J. Murcia-García* \\ *Servicio de Neurocirugía y Unidad de Neurocirugía Pediátrica. Hospital Universitario "Virgen de la Arrixaca". Murcia. **Laboratorio de \\ Neurología y Neurocirugía Experimental. Facultad de Medicina. Universidad de Murcia. ***Sección de Anestesia Pediátrica. Hospital Infantil \\ Universitario "Virgen de la Arrixaca". Murcia
}

Resumen

Antecedentes. La médula espinal puede fijarse tras la reparación inicial en pacientes operados de mielomeningocele (MMC) y lipomeningocele (LMC), produciendo graves lesiones físicas y psicológicas. Asimismo, la introducción accidental de restos cutáneos durante la reparación de estas lesiones puede dar lugar al desarrollo de tumores intraespinales de estirpe cutánea.

Objetivos. Averiguar la incidencia del anclaje medular tras la cirugía de MMC y LMC y analizar los factores que puedan explicar su aparición. También, investigamos las maniobras técnicas durante la operación primaria susceptibles de evitar la aparición del síndrome de médula fijada.

Pacientes y métodos. Revisión retrospectiva de las historias de los pacientes operados de MMC $(n=162)$ y de espina bífida oculta $(n=54)$ en el período 1975-2005 que desarrollaron cuadros de anclaje medular sintomático.

Resultados. Once pacientes con MMC (6,79\%) y dos con LMC (3,7\%) presentaron manifestaciones de anclaje medular tras intervalos de 2 a 37 años después de la reparación primaria. Las indicaciones de reintervención se basaron fundamentalmente en criterios clínicos. Un hallazgo constante fue la fibrosis cicatricial que estuvo presente en todos los casos. Otros hallazgos operatorios causantes de la fijación medular consistieron en tumores cutáneos de inclusión $(\mathrm{n}=\mathbf{3})$, reacciones de cuerpo extraño $(n=2)$, estenosis del canal lumbar $(n=2)$, restos de lipoma $(n=1)$, y filum hialinizado (n=1). En 3 casos de MMC se encontró además un quiste epidermoide intradural $(1,85 \%$ de los MMC), lo que supone una tasa de epidermoides en la reintervención de MMC de $\mathbf{2 7 \%}$. El periodo medio de seguimiento fue de 5,5 años y los resultados fueron: mejoría en 8, dos no experimentaron cambios, y uno sufrió empeoramiento.

Recibido: 20-04-06. Aceptado: 15-05-06
Conclusiones. El deterioro neurológico de los pacientes operados de MMC o LMC no constituye una consecuencia obligada o parte de la historia natural de estos procesos. EI deterioro, precoz o tardío, puede estar motivado por el anclaje posquirúrgico de la médula espinal. Ello hace necesario realizar un seguimiento periódico de estos pacientes, acompañado de los oportunos estudios de neuroimagen y, en su caso, de exploración quirúrgica. Los resultados fueron satisfactorios, ya que el $92 \%$ de los pacientes reoperados experimentaron mejoría o estabilización de su enfermedad. Se describen además dos causas infrecuentemente descritas de deterioro tardío: la estenosis del canal y las reacciones fibrosas de cuerpo extraño a materiales implantados.

PALABRAS CLAVE: Anclaje medular. Epidermoide intraespinal. Espina bífida. Estenosis del canal lumbar. Lipomeningocele. Médula anclada. Mielomeningocele. Reacción de cuerpo extraño.

Spinal cord tethering in myelomeningocele and lipomeningocele patients: the second operation

Summary

Background. Spinal cord rethetering can occur after the primary surgical repair of myelomeningoceles (MMC) and lipomeningoceles (LMC) and produce devastating physical and psychological consequences. The inadvertent introduction of skin elements at the time of the initial surgery can lead to the growth of intraspinal epidermoid or dermoid cysts.

Objectives. To review the incidence of spinal cord tethering following surgery for open and occult spinal dysraphism and to analyze factors that might influence the appearance of this complication. We also aimed to search technical measures at the time of the primary operation that might prevent the occurrence of symptomatic cord retethering.

Material and methods. We reviewed the medical records of patients submitted to surgical repair of MMC 
$(n=162)$ or occult spinal dysraphism $(n=54)$ during the period 1975-2005 who developed symptomatic tethered cord syndrome.

Results. Eleven of $162(6.79 \%)$ patients with MMC and 2 of $54(3.7 \%)$ with LMC developed clinical symptoms and signs of spinal cord tethering after intervals ranging from 2 to 37 years after the initial surgical repair of their back lesions. Indications for surgical re-exploration were based mainly on clinical grounds. Postoperative fibrosis was a constant finding in all instances. Other surgical findings included inclusion tumors of cutaneous origin $(n=3)$, lumbar canal stenosis $(n=2)$, foreign body reactions $(n=2)$, residual lipoma $(n=1)$, and a tight hyalinized filum $(n=1)$. Interestingly, 3 of 162 (or $1.85 \%$ ) myelomeningoceles were found to harbor an intradural epidermoid tumor at the time of spinal cord dethetering, accounting for an incidence of cutaneous inclusion tumors of $27 \%$ in cases of postMMC repair tethering. After a mean follow-up time of 5.5 years, eight patients were improved, two were unchanged and one was worsened.

Conclusions. Neurological deterioration is not a necessary consequence of the natural history of patients with MMC or LMC. Early or late clinical deterioration can be due to spinal cord re-tethering and deserves timely investigation and surgical exploration. Results of surgical intervention were rewarding as $92 \%$ of the patients showed improvement or stabilization in their otherwise deteriorating condition. We also report two infrequent causes of spinal cord deterioration: lumbar canal stenosis and intense foreign-body reactions to implanted materials.

KEY WORDS: Intraspinal epidermoid. Lipomeningocele. Lumbar canal stenosis. Myelomeningocele. Postsurgical foreign-body reaction. Spina bifida. Tethered cord.

\section{Introducción}

El síndrome de anclaje medular es una condición patológica que se asocia habitualmente con malformaciones espinales como el mielomeningocele (MMC), lipomeningocele (LMC), engrosamiento del filum terminale, diastematomielia, y lipoma intradural. Otras causas responsables del síndrome son los tumores dermoides y epidermoides congénitos o adquiridos y la aracnoiditis $2^{2,9,10}$. El diagnóstico se establece por la combinación de (i) síntomas y signos neurológicos de afectación medular y radicular y (ii) la presencia de un cono medular situado por debajo del nivel vertebral L2 en estudios de neuroimagen ${ }^{9,10,24}$. E1 mecanismo fisiopatológico que explica las manifestaciones clínicas es el daño inflingido a la médula por factores vasculares, metabólicos o mecánicos, cuando ésta se ve sometida a tracción por una de las lesiones anteriormente mencionadas ${ }^{30}$.

Los objetivos de este trabajo fueron: (i) analizar la incidencia del síndrome de re-anclaje medular en una población de pacientes operados de MMC o LMC y, (ii) revisar las causas que podrían contribuir al desarrollo de este síndrome con la finalidad de identificar posibles medidas de prevención. Describimos además, dos causas infrecuentes que pueden ocasionar anclaje medular: la estenosis congénita del canal lumbar asociada a espina bífida y las reacciones de cuerpo extraño producidas por los materiales implantados.

\section{Pacientes y métodos}

Se revisaron las historias clínicas de los pacientes operados de MMC o LMC durante el período 1975-2005. Durante este tiempo se realizaron 162 cierres quirúrgicos de MMCs y 54 operaciones de liberación microquirúrgica de espina bífida oculta. Los criterios de exclusión consistieron en (i) casos que no aceptaron reintervención ( $\mathrm{n}=1)$, (ii) pacientes operados en otros servicios $(n=1)$ y (iii) casos operados de MMC que presentaban anclaje en las pruebas de neuroimagen pero que estaban asintomáticos. También se incluyeron dos pacientes operados inicialmente en otros centros y que fueron remitidos a nuestro Servicio tras la aparición de síntomas de anclaje. Se registraron los datos clínicos y epidemiológicos referentes a la edad a la reintervención, sexo, intervalo desde la operación inicial a la intervención de anclaje medular, síntomas y signos indicativos del deterioro, resultados de los estudios complementarios de neuroimagen, así como los protocolos quirúrgicos de la primera operación $(\mathrm{n}=11)$ y las notas operatorias de las reintervenciones. Igualmente se anotaron las complicaciones de la cirugía. Los resultados y el tiempo de seguimiento se obtuvieron de las hojas de evolución de la Consulta Externa. Los resultados se clasificaron en 3 grupos: mejor, igual o peor, según la evolución postoperatoria de las manifestaciones clínicas en comparación con el estado prequirúrgico. No hubo casos de pérdida de seguimiento.

\section{Resultados}

Incidencia de reanclaje en MMC y LMC. En el periodo comprendido de 1975 a 2005 se han operado 162 MMCs, de los que 11 desarrollaron síntomas de anclaje medular, con una incidencia de 6,8\%. Durante el mismo tiempo se intervinieron 54 pacientes con espina bífida oculta, casi todos con LMC, de los que 2 presentaron cuadros de reanclaje medular sintomático, con una incidencia de 3,7\%.

Datos epidemiológicos y clínicos (Tabla 1). Cuatro de los 13 pacientes eran varones y 9 mujeres. La edad mediana de presentación con síntomas de anclaje medular fue lige- 
Tabla 1

Datos epidemiológicos y clínicos de 13 pacientes con anclaje medular postoperatorio

\begin{tabular}{|c|c|c|c|}
\hline & & Mielomeningocele & Lipomeningocele \\
\hline \multirow[t]{8}{*}{ Datos epidemiológicos } & Sexo M/F & $4 / 7$ & $0 / 2$ \\
\hline & Edad (mediana) (rango) & 7 años (2-37) & 21,5 años $(16-27)$ \\
\hline & Intervalo & 7 años $(2-37)$ & 16,5 años $(16-17$ \\
\hline & Hidrocefalia & 6 & - \\
\hline & Chiari II & 9 & - \\
\hline & Siringomielia & 3 & - \\
\hline & Dolor & 8 & 2 \\
\hline & Déficit progresivo & 10 & 2 \\
\hline \multirow[t]{5}{*}{ Hallazgos quirúrgicos } & Fibrosis & 11 & 2 \\
\hline & Anclaje filum & 6 & 1 \\
\hline & Reacción c. extraño & 1 & 1 \\
\hline & Epidermoide/dermoide & 3 & - \\
\hline & Estenosis canal lumbar & 2 & - \\
\hline \multirow[t]{3}{*}{ Resultados } & Mejor & 8 & 2 \\
\hline & Igual & 2 & - \\
\hline & Peor & 1 & - \\
\hline \multirow[t]{3}{*}{ Complicaciones } & Fístula LCR con infección profunda (reop.) & 1 & - \\
\hline & Nuevo reanclaje (reop.) & - & 1 \\
\hline & Infección & 1 & - \\
\hline
\end{tabular}

$\mathrm{M}=$ masculino, $\mathrm{F}=$ femenino, reop.=reoperación

ramente menor (7 años) para los niños operados de $\mathrm{MMC}$, sin embargo, el rango de edad fue muy variable de 2 a 37 años. En el subgrupo de LMC, la edad de presentación fue de 21,5 (rango de 16 a 27) años. El intervalo entre la operación original y la aparición de las manifestaciones de anclaje medular fue de 7 años para los operados de MMC y de 16,5 años para los intervenidos de LMC. Los pacientes operados de MMC lo habían sido en el período neonatal y las dos niñas con LMC habían sido intervenidas a los 3 meses y a los 10 años respectivamente. Las manifestaciones clínicas de presentación consistieron en empeoramiento motor $(n=12)$, dolor lumbar o radicular $(n=10)$, claudicación intermitente $(n=5)$, parestesias $(n=4)$, aparición o empeoramiento de deformidad ortopédica $(n=4)$, empeoramiento de la función vesical $(n=4)$, úlceras en los pies de cicatrización tórpida $(n=3), y$ actitud en flexión de las piernas al caminar $(\mathrm{n}=2)$.

El diagnóstico se realizó mediante la comparación de los hallazgos clínicos de la exploración más reciente con los exámenes neurológicos previos que constaban en la historia de consultas externas. La presencia del anclaje se confirmó con Resonancia Magnética (RM) en 10 pacientes y mediante mielografía en 1 (Figs. 1-4). Se realizaron pruebas neurofisiológicas en 8 casos. En siete pacientes con hidrocefalia se comprobó la correcta función de la válvula mediante RM cerebral o punción del reservorio. Asimismo, en la RM se comprobó la presencia de malformación de Chiari tipo II en 9 pacientes ( 8 con MMC y 1 con LMC). Tres pacientes presentaban siringomielia localizada en un caso en región cervico-dorsal y en dos en el cono medular. 
Neurocirugía

Reanclaje medular en pacientes con mielomeningocele y lipomeningocele: la segunda operación

2007; 18: 312-319

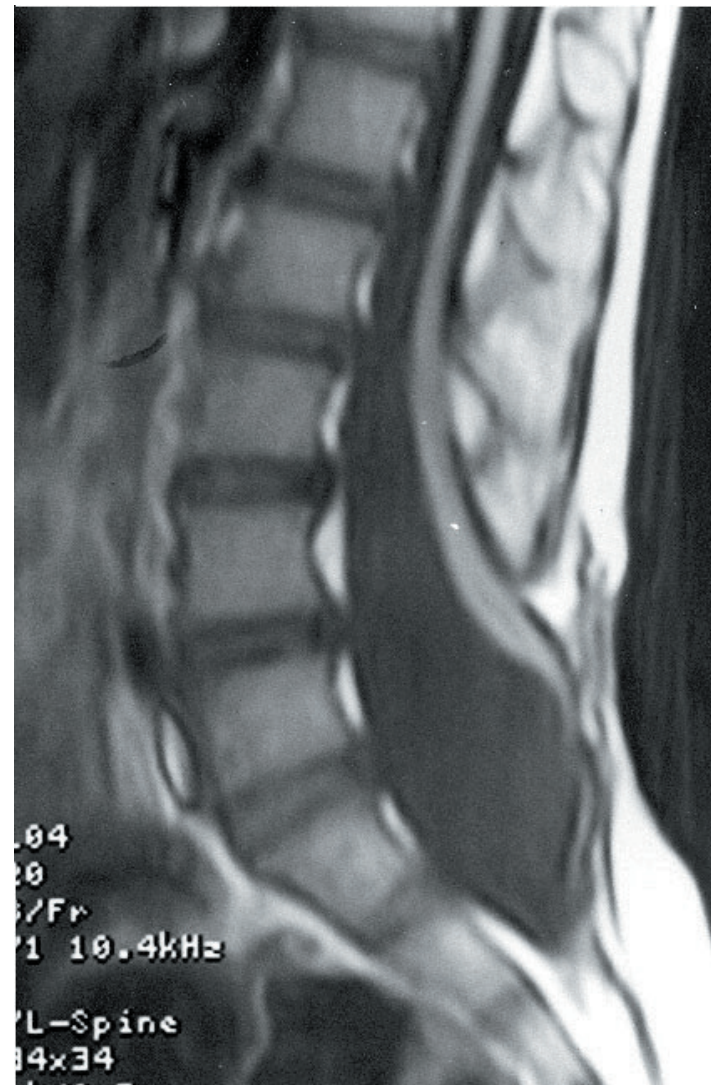

Figura 1. RM: imagen típica de anclaje

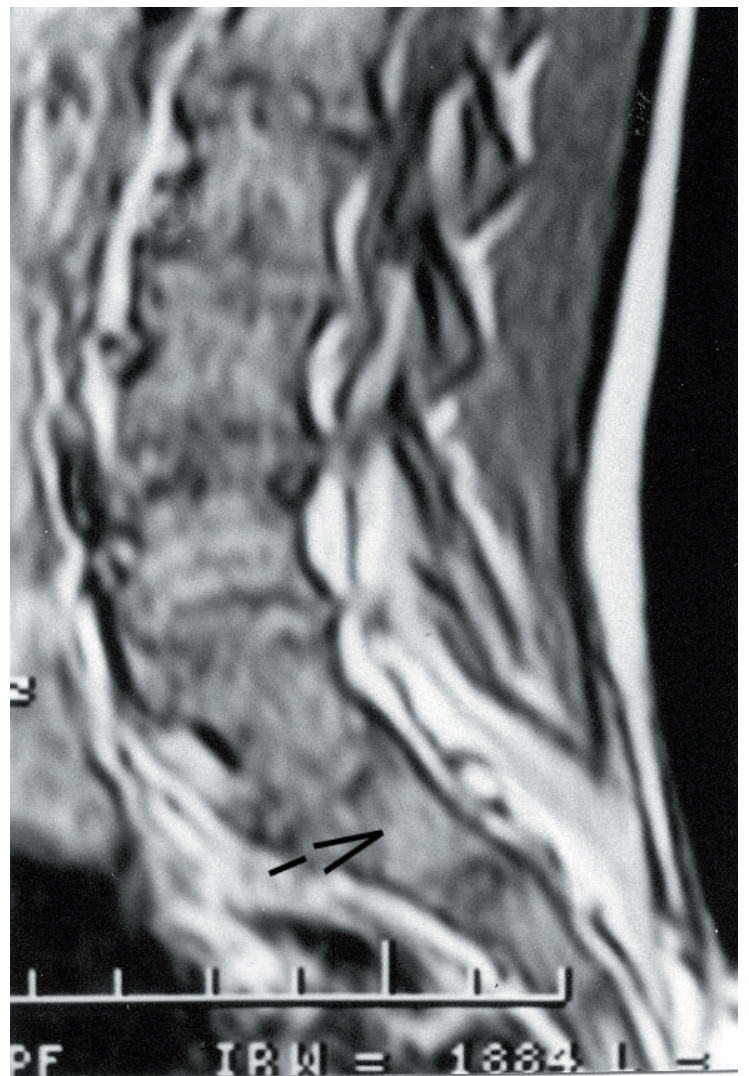

Figura 2. RM: epidermoide en MMC (flecha)
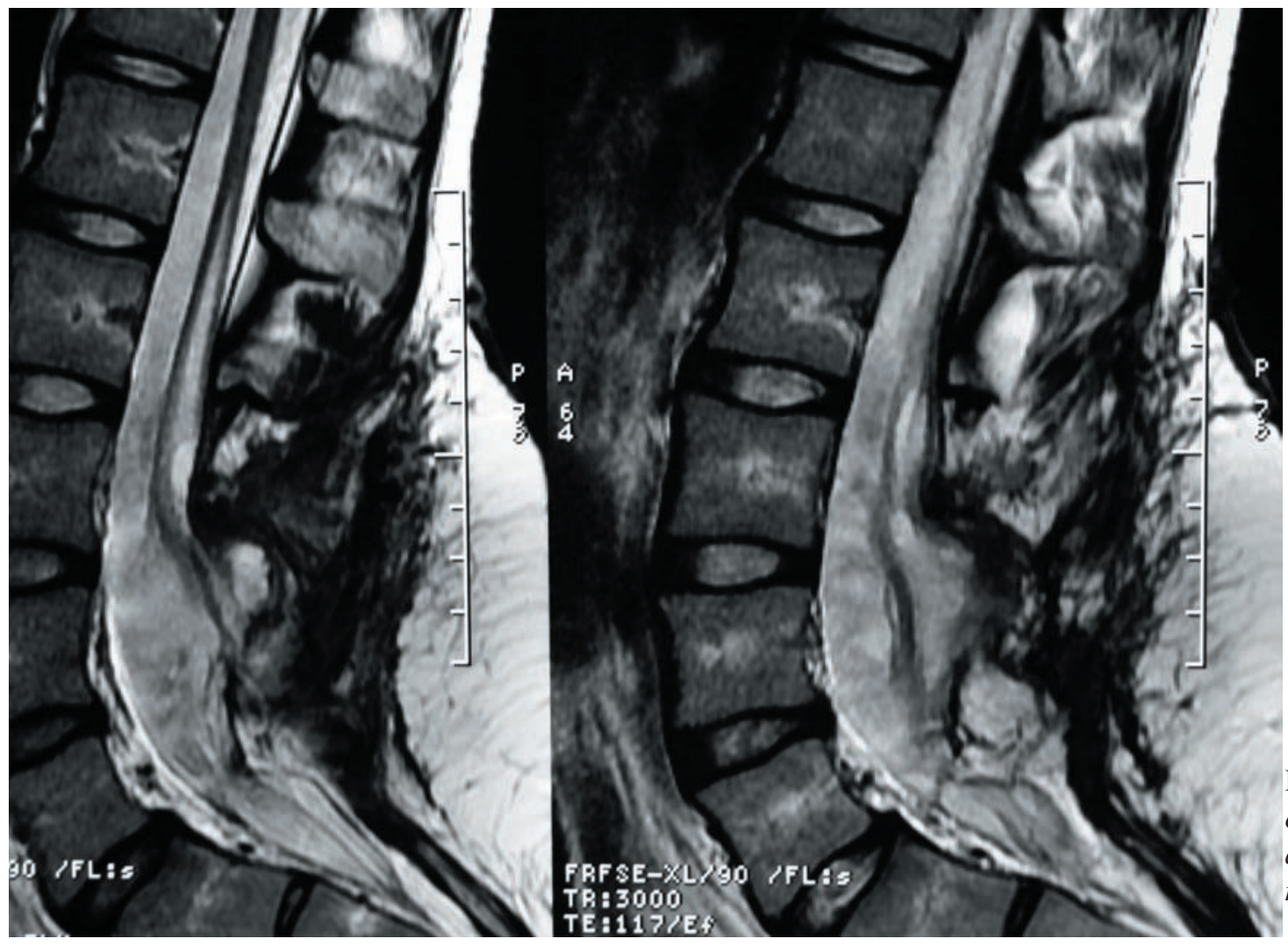

Figura 3. $R M$ en una niña con anclaje postreparación de $L M C$ 


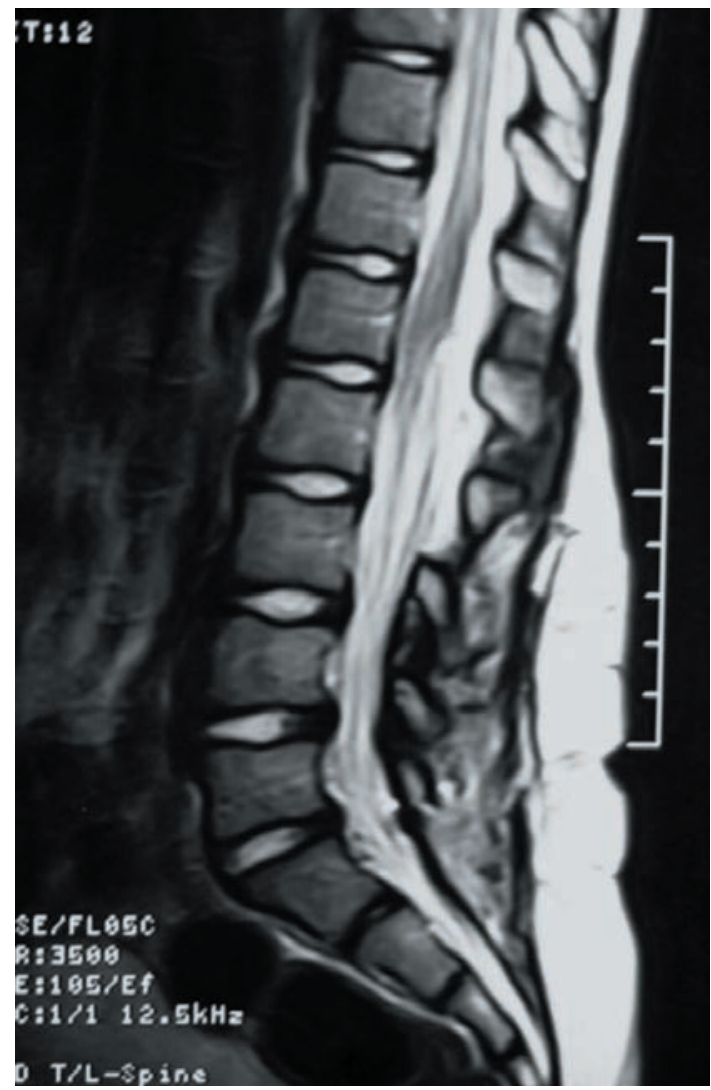

Figura 4. Estenosis del canal lumbar en MMC

Hallazgos operatorios. Las técnicas quirúrgicas empleadas en la operación inicial son las habituales que se usan para la reparación quirúrgica del MMC y para descompresión de los $\operatorname{LMCs}^{1,5,19,28}$. El cierre de la duramadre en la primera intervención fue directo en 10 ocasiones, habiéndose utilizado plastias en tres casos, dos con fascia lumbar antóloga y una con duramadre liofilizada. Por definición la médula estaba fijada a la altura de la lesión inicial (L5-Sacro en 12

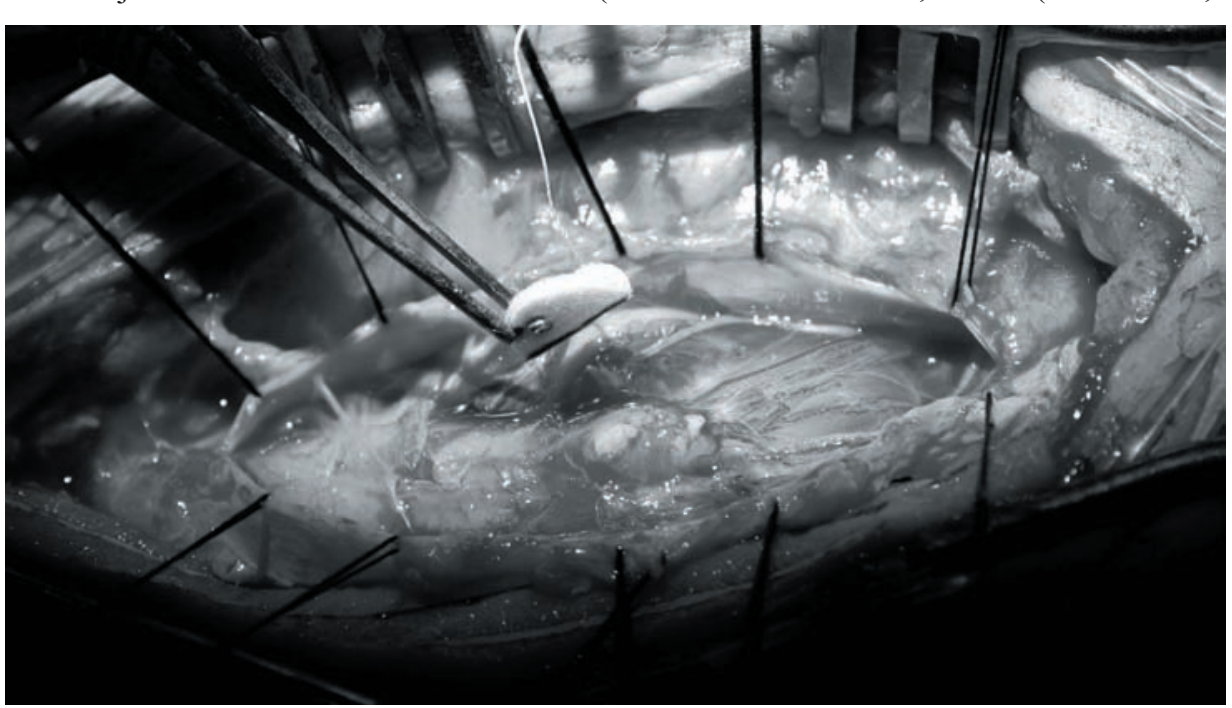

y L3-L4 en 1 caso). Las adherencias estaban constituidas por tejido fibroso cicatricial que inmovilizaba a la médula en una extensión que osciló de 1 a 3 segmentos (Fig. 1). En siete pacientes el filum terminale estaba engrosado y fue seccionado. En uno de estos casos, el filum era más rígido de lo habitual y la anatomía patológica mostró una marcada hialinización. Tres pacientes con MMC tenían tumores epidermoides intradurales, uno entre las raíces de la cola de caballo, y dos en la cara dorsal de la médula (Fig. 2 y 5). En dos pacientes había una estenosis del canal lumbar en la última vértebra no afectada que contribuía a la compresión medular (Fig. 4). En el caso que había recibido una plastia de duramadre liofilizada las adherencias a la médula eran particularmente densas. En una niña operada de MMC la tracción medular estaba causada por una intensa reacción de cuerpo extraño alrededor de una sutura de seda utilizada en la reparación inicial, presentando el aspecto de una fibrosis que incluía al hilo de seda estirado longitudinalmente. En otra paciente tratada de LMC se descomprimió con láser un resto de lipoma que podría contribuir también al anclaje (Fig. 3). En dos pacientes con estenosis lumbar se practicó laminectomía bilateral completa de la última vértebra sana ${ }^{4,16,27}$.

Complicaciones. Una paciente con LMC presentó una grave reacción alérgica al látex durante la inducción anestésica que obligó a diferir la operación. Las complicaciones postoperatorias consistieron en: una infección superficial de la herida tratada con medios tópicos, una infección profunda (Escherichia Coli y Corynebacterium Sp.) por fístula de LCR, que requirió reintervención, y un seroma que curó con tratamiento conservador. Una paciente con reanclaje post-LMC volvió a empeorar y fue el único caso que requirió una nueva exploración quirúrgica por recidiva de los síntomas al año de la reintervención.

Resultados. El tiempo medio de seguimiento fue de 5,5 años (extremos 0,5 a 9). El dolor desapareció tras la
Figura 5. Detalle de la operación de anclaje medular mostrando un epidermoide en la cara dorsal de la médula 
operación en todos los pacientes. Los resultados funcionales fueron: mejoría en 8 , detención del deterioro en 2 , y empeoramiento leve en 1 caso. Una paciente con MMC reintervenida a los 37 años y otro paciente con MMC operada a los 6 años no experimentaron ninguna mejoría. El paciente que empeoró tenía un tumor epidermoide con salida de contenido y fuertes adherencias entre las raíces de la cauda equina.

\section{Discusión}

Datos epidemiológicos. La incidencia de MMC es de aproximadamente 0,5 por 1000 nacidos vivos en España y países de nuestro entorno. El MMC produce secuelas permanentes como pérdida de función motora y sensorial en las extremidades inferiores, incontinencia vesical y anal, y se acompaña de anomalías esqueléticas, hidrocefalia y malformación de Chiari II. El LMC es una variedad de la espina bífida oculta y una de las principales causas de anclaje medular. Las manifestaciones del LMC son atribuidas a la tracción o isquemia de los segmentos inferiores de la médula y cono medular ${ }^{30}$, e incluyen déficit motor, espasticidad, alteraciones sensitivas, disfunción de esfínteres y deformi-

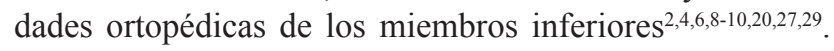
La mayoría de niños operados de MMC, y algunos niños tras la cirugía del LMC, presentan fijación de la médula espinal por adherencias de la duramadre en el nivel de la reparación quirúrgica inicial. Cuando se produce este contacto entre la médula y la duramadre, la parte caudal de la médula y el cono medular pueden quedar atrapados por el tejido cicatricial ${ }^{2,4,6,8-10,20,27,29}$. La incidencia del síndrome de anclaje medular oscila entre el $3 \%$ al $20 \%$ de los pacientes operados de MMC o LMC en diversas publicaciones ${ }^{2,4,6,8-}$ 10,20,24,27,29. Nuestra incidencia de re-anclaje fue de $6,8 \%$ en el caso de MMCs y de 3,7\% para los casos de LMC. Esta baja tasa de cuadros de reanclaje probablemente es real, ya que la mayoría de los pacientes operados de espina bífida son seguidos regularmente en nuestra Consulta Externa.

El intervalo medio hasta la presentación fue de 7 años para los MMCs, aunque en un caso el deterioro se produjo muy precozmente, a los 2 años de la operación. Otra paciente fue remitida a Consulta a la edad de 37 años. Se ha descrito una mayor incidencia de deterioro en los períodos de crecimiento rápido, que en nuestra serie fue alrededor de los 13 años. Existe un predominio claro de mujeres (9/13 casos), sin que tengamos ninguna explicación para este hecho. En la mayoría de los casos el cuadro del re-anclaje medular se produjo en los segmentos más inferiores de la médula, es decir a nivel lumbosacro, como sucedió en 12 de nuestros pacientes.

Diagnóstico. Las manifestaciones clínicas del síndrome de anclaje medular incluyen aumento del déficit motor (55\%), empeoramiento de la marcha (54\%), escoliosis
(51\%), dolor lumbar o radicular $(32 \%)$, aparición de nuevas deformidades ortopédicas (11\%) y empeoramiento de los esfínteres $(6 \%)^{2,4,6,8-10,20,27,29}$. Los síntomas y signos de presentación del cuadro de anclaje en nuestros pacientes fueron similares a los descritos en diferentes trabajos (Tabla 1) 2,4,6,8-10,20,27,29. En niños operados de LMC, que tienen habitualmente una situación neurológica mejor que los tratados de $\mathrm{MMC}$, el empeoramiento clínico es detectado más fácilmente, por lo que son referidos a la consulta más precozmente. Por el contrario, el deterioro de los pacientes con MMC es notado más tardíamente ya que, con frecuencia, pasa desapercibido o es atribuido erróneamente a la historia natural de la enfermedad. El diagnóstico del re-anclaje medular se basa fundamentalmente en datos clínicos $^{9,10}$. Los estudios de neuroimagen son de utilidad para valorar la extensión del anclaje y para el despistaje de lesiones asociadas que puedan contribuir a la fijación o compresión de la médula, como diastematomielia, quistes aracnoideos y tumores epidermoides fundamentalmente ${ }^{12}$. Los estudios neurofisiológicos, como la electromiografía y los potenciales evocados sólo son de utilidad cuando se dispone de registros previos que permiten una comparación ${ }^{3}$. De la misma forma se ha destacado en varios estudios la importancia de los registros urodinámicos y su comparación con estudios anteriores al deterioro 9 . Recientemente se emplea la ecografía para determinar el flujo de LCR en la zona lesionada ${ }^{2}$.

El diagnóstico diferencial del cuadro de anclaje medular en niños operados de MMC se debe de realizar con la presencia de hidrocefalia no tratada, malfunción valvular, anomalía de Chiari II o siringomielia sintomáticas ${ }^{4,12,24}$.

Tratamiento quirúrgico, complicaciones y prevención. El cierre inicial del MMC consiste en el aislamiento de la placa neural del tejido cutáneo circundante (para evitar la formación de epidermoides de inclusión) y su reposición dentro del saco dural, lo que en la mayoría de los casos se lleva a cabo sin dificultad ${ }^{1,5,19,28}$. La sutura de la duramadre debe ser hermética para evitar fístulas de LCR, pero evitando comprimir el tejido neural. Algunos autores recomiendan el uso de suturas absorbibles para disminuir las posibilidades de reacción de cuerpo extraño. En uno de nuestros pacientes la causa del reanclaje era una fibrosis intensa que tenía como eje un hilo de seda dispuesto longitudinalmente a todo lo largo de la zona de sutura dural. Se debe intentar por todos los medios el recubrimiento de la meninge con fascia y/o musculatura lumbosacra.

En el caso del LMC se procurará extirpar el máximo posible de tejido lipomatoso, pero sin intención de ser exhaustivos, ya que es preferible dejar una capa de lipoma sobre la médula a arriesgar la integridad funcional del tejido medular ${ }^{10}$. La exposición quirúrgica debe ser lo suficientemente amplia como para explorar la médula craneal y caudalmente, así como a ambos lados de la entrada del tallo lipomatoso, de manera que al final de la operación 
haya circulación libre de LCR alrededor de la médula. En este momento se examina el filum y, si parece indicado, se secciona. El objetivo fundamental de la operación del LMC es liberar el tejido neural que debe quedar "flotando" libremente en el LCR. En caso necesario, se aumenta la capacidad del saco dural mediante una plastia, siendo casi siempre factible la obtención de un trozo de fascia autóloga para tal fin, evitando la colocación de sustitutos durales, biológicos o sintéticos, por los riesgos que comportan ${ }^{15,17}$. Aunque se ha utilizado silastic o Gore-Tex ${ }^{11,28}$, ningún sustituto dural, hasta ahora, parece que pueda evitar la formación de adherencias ${ }^{6}$.

En cuanto a la operación para el tratamiento del reanclaje, los principios son similares a los descritos para la reparación del $\mathrm{LMC}^{8,10}$, empezando la exploración quirúrgica desde tejido sano, tanto por la parte craneal como por la caudal, siguiendo una técnica de liberación circunferencial ${ }^{18}$. En la reintervención se liberan las adherencias mediante disección con bisturí o microtijeras y se explora de manera sistemática la presencia de otros elementos que pudieran contribuir a la fijación o compresión de la médula, como bandas cicatriciales, filum corto o engrosado, y tumores epidermoides o dermoides. Igualmente se debe considerar la laminectomía descompresiva cuando la estenosis del canal lumbar parece ser un factor causal ${ }^{8,16,27}$.

Respecto a los resultados y complicaciones quirúrgicas nuestros datos son similares a los ya publicados ${ }^{2,10,24,27,29}$. Un $70-90 \%$ de los pacientes mejoran o experimentan una

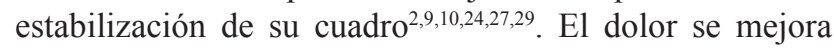
prácticamente en todos los casos, los síntomas motores en el 50\% y los de los esfínteres en el 15\% aproximadamente $^{29}$. Phuong et al., de la Clínica Mayo, han revisado la historia natural de los casos de anclaje post-MMC ${ }^{20}$. De un total de 1435 niños con MMC, 45 pacientes con cuadro de anclaje medular no fueron reintervenidos, recibiendo en cambio tratamiento sintomático como ampliación vesical, tratamiento de la espasticidad, etc. ${ }^{20}$. La incidencia de los síntomas de anclaje, para estos autores, fue del 27,5\% al año, del $40 \%$ a los 2 años y del $60 \%$ a los 5 años $^{20}$. Esto implica un apoyo importante para indicar la operación de desanclaje cuando aparecen los síntomas iniciales ${ }^{20}$.

Tumores dermoides y epidermoides. Un aspecto interesante en la patogenia del síndrome del re-anclaje medular en niños operados de MMC es el hallazgo de tumores dermoides o epidermoides en la zona de la reintervención ${ }^{1,13,23}$. En nuestra casuística de 162 casos de espina bífida abierta hemos encontrado sólo 1 paciente con un epidermoide congénito adherido a la placa neural descubierto al efectuar el cierre quirúrgico de un meningocele lumbosacro, lo que supone una incidencia de $0,61 \%$ de epidermoide congénitos asociados a $\mathrm{MMC}^{14,22}$. Por el contrario, hemos hallado 3 casos de epidermoides intradurales en casos de reintervención, lo que implica una incidencia de
3,7\% de todos los MMCs operados y del 27\% de los casos de re-anclaje medular post-MMC. Mazzola et al. han descrito recientemente la aparición de dermoides de inclusión en casos de reparación intrauterina del MMC que atribuyen al implante de piel y al uso de duraplastia con dermis acelular humana, que favorecería el crecimiento intradural de células dérmicas o epidérmicas ${ }^{18}$. Sin embargo no tuvimos casos de tumores de inclusión tras la cirugía de los LMC $(p=0,049)$, aunque recientemente se ha descrito un caso de esta complicación $^{25}$. Estos tumores probablemente proceden de la inclusión de pequeños restos epidérmicos en el momento de la reparación quirúrgica inicial del $\mathrm{MMC}^{1,9,13,23}$. No obstante, algunos autores refieren que, con frecuencia, existen inclusiones de tejido epidérmico en el estudio histológico de la placa neural, que podrían justificar la ulterior aparición espontánea de estos tumores de origen epitelial ${ }^{26}$. La hipótesis de que los epidermoides intradurales se deben a la introducción accidental, durante el cierre del MMC, de pequeños fragmentos cutáneos, indicaría la conveniencia de utilizar magnificación de manera rutinaria en la intervención inicial del MMC para evitar esta complicación ${ }^{1}$.

Reacciones de cuerpo extraño. Dos casos de nuestra serie merecen especial consideración por sus implicaciones en la prevención del cuadro de re-anclaje medular. En uno de ellos, la fijación medular estaba producida por una reacción de cuerpo extraño alrededor de la sutura de seda continua utilizada al cerrar el MMC. En el otro, la reacción fibrosa, muy intensa, parecía estar provocada por la migración intradural del trozo de plastia de duramadre liofilizada. Este tipo de respuesta biológica al implante de cuerpos extraños ya ha sido comentada en alguna publicación $^{7,21}$. El interés de estas observaciones radicaría en la recomendación de evitar utilizar plastias durales de tejidos biológicos o sintéticos, empleando, en su lugar, una pieza de fascia lumbar adyacente ${ }^{17}$. Asimismo, el material de sutura de la duramadre puede ser reabsorbible, evitando así la utilización de hilos de seda ${ }^{10}$.

\section{Conclusiones}

Hemos revisado las historias de 13 pacientes con cuadros de anclaje medular tras operaciones de MMC o LMC. Los síntomas de presentación fundamentalmente consistieron en dolor y empeoramiento motor, sensorial y de los esfínteres, así como cuadros de claudicación intermitente. El diagnóstico es fundamentalmente clínico, tratándose básicamente de un déficit neurológico nuevo que se añade a un déficit neurológico pre-existente. Se comentan los hallazgos operatorios y se destacan aquellas medidas que podrían evitar la aparición de la fibrosis y la formación de epidermoides intradurales. Como medidas quirúrgicas, se recomienda la disección cuidadosa de la placa neural para impedir el implante de restos de piel, y evitar el uso de 
materiales extraños (duraplastia y suturas de seda). Se destaca la importancia de la revisión periódica de estos pacientes para la detección precoz del cuadro de anclaje medular.

\section{Bibliografía}

1. Amacher, L.: The microsurgical anatomy of lumbar rachischisis. Adv Ophthal 1978; 37: 197-202.

2. Balasubramaniam, C., Laurent, J.P., McCluggage, C., Oshman, D., Cheek, W.R.: Tethered cord syndrome after repair of myelomeningocele. Childs Nerv Syst 1990; 6: 208-211.

3. Boor, R., Schwarz, M., Reitter, B., Voth, D.: Tethered cord after spina bifida aperta: a longitudinal study of somatosensory evoked potentials. Childs Nerv Syst 1993; 9: 328-330.

4. Caldarelli, M., Di Rocco, C., Colosimo, C. Jr., Fariello, G., Di Gennaro, M.: Surgical treatment of late neurological deterioration in children with myelodysplasia. Acta Neurochir (Wien) 1995; 137: 196-206.

5. Cheek, W.R., Laurent, J.P., Cech, D.A.: Operative repair of lumbosacral myelomeningocele (technical note). J Neurosurg 1983; 59: 718-722.

6. Colak, A., Pollack, I.F., Albright, A.L.: Recurrent tethering: common long-term problem after lypomyelomeningocele repair. Pediatr Neurosurg 1998; 29: 184-190.

7. Cole, J.S. IV, Pittman, T.: Intradural mesh: an unusual cause of spinal cord tethering (Case report). J Neurosurg 2005 (Pediatrics 3); 102: 311-313.

8. Di Rocco, C., Peter, J.C.: Management of tethered spinal cord. Surg Neurol 1997; 48: 320-322.

9. Herman, J.M., McLone, D.G., Store, B.B., Dauser, R.C.: Analysis of 153 patients with myelomeningocele or spinal lipoma reoperated upon for a tethered cord. Pediatr Neurosurg 1993; 19: 234-239.

10. Hudgings, R.G., Gilreath, C.L.: Tethered spinal cord following repair of myelomeningocele. Neurosurg Focus 2004; 16 (2) Article 7, pp 1-4.

11. Inoue, H.K., Kobayashi, S., Ohbayashi, K., Kohga, H.: Treatment and prevention of tethered and retethered cord using a Gore-Tex surgical membrane. J Neurosurg 1994; 80:689-693.

12. Just, M., Schwartz, M., Ludwig, B., Ermert, J.: Cerebral and spinal MR-findings in patients with post-repair myelomeningocele. Pediatr Radiol 1990; 20: 262-266.

13. Kirsch, W.M., Hodges, F.J.: An intramedullary epidermal inclusion cyst of the thoracic cord associated with a previously repaired meningocele. J Neurosurg 1966; 24: 1018-1020.

14. Martínez-Lage, J.F., Masegosa, J., Sola, J., Poza M.: Epidermoid cyst occurring within a lumbosacral myelomeningocele. J Neurosurg 1983; 59: 1095-1097.

15. Martínez-Lage, J.F., Poza, M., Sola, et al.: Accidental transmission of Creutzfeldt-Jakob disease by dural cadaveric grafts. J Neurol Neurosurg Psychiatry 1994; 57: 1091-1094.

16. Martínez-Lage, J.F., Piqueras, C., Poza, M.: Lumbar canal stenosis. A cause of late neurological deterioration in patients with spina bifida. Surg Neurol 2001; 55: 256-260.

17. Martínez-Lage, J.F., Pérez-Espejo, M.A., Palazón, J.H., Lopez Hernández, F., Puerta, P.: Autologous tissues for dural grafting in children: a report of 56 cases. Childs Nerv Syst 2006; 22: 139-144.

18. Mazzola, C.A., Albright, A.L., Sutton, L.N., Tuite, G.F., Hamilton, R.L., Pollack, I.F.: Dermoid inclusion cysts and early spinal cord tethering after fetal surgery for myelomeningocele. N Engl J Med 2002; 347: 256-259.

19. McLone, D.G.: Technique for closure of myelomeningocele. Child's Brain 1980; 6: 65-73.

20. Phuong, L.K., Schuberl, K.A., Raffel, C.: Natural history of tethered cord in patients with myelomeningocele. Neurosurgery 2002; 50: 989-995.

21. Rossotch, E. Jr., Bullard, D.E., Oakes, W.K.: Delayed foreign-body reaction to silk sutures in pediatric neurosurgical patients. Childs Nerv Syst 1987; 3: 375-378.

22. Sakai, K., Sakamoto, K, Kobayashi, N., Iguchi, H.: Dermoid cyst within an upper thoracic meningocele. Surg Neurol 1996; 45: 287-292.

23. Scott, R.M., Wolpert, S.M., Batoshesky, L.E., Zimbler, S., Klauber, G.T.: Dermoid tumors occurring at the site of previous myelomeningocele repair. J Neurosurg 1986; 65:779-783.

24. Shurtleff, D.B., Duguay, S., Duguay, G., et al.: Epidemiology of tethered cord with myelomeningocele. Eur J Pediatr Surg 1997; 7 (Suppl I): 7-11.

25. Song, J.H., Kim, M.H., Shin, K.M.: Intraspinal epidermoid cyst occurring 15 years alter lipomyelomeningocele repair (Case report). J Neurosurg (Spine 2) 1999; 90: 252-254.

26. Storrs, B.B.: Are dermoid and epidermoid tumors preventable complications of myelomeningocele repair? Pediatr Neurosurg 1994; 20: 160-162.

27. Tamaki, N., Shirataki, K., Kojima, N., Shouse Y, Matsumoto, S.: Tethered cord syndrome of delayed onset following repair of myelomeningocele. J Neurosurg 1988; 69:393-398.

28. Venes, J.L.: Surgical consideration in the initial repair of myelomeningocele and the introduction of a technical modification. Neurosurgery 1985; 17: 111-113.

29. Villarejo, F.J.: Médula anclada: reanclaje por mielomeningocele. En Villarejo, F., Martínez-Lage, J.F. (eds): Neurocirugía Pediátrica. Ergon, Madrid, 2001; pp. 191.

30. Yamada, S., Zinke, D.E., Saunders, D.: Pathophysiology of tethered cord syndrome. J Neurosurg 1981; 54:494-503

Martínez-Lage, J.F.; Ruiz-Espejo Vilar, A.; Almagro, M.J.; Sánchez del Rincón, I.; Ros de San Pedro, J.; FelipeMurcia, M.; Murcia-García, F.J.: Reanclaje medular en pacientes con mielomeningocele y lipomeningocele: la segunda operación. Neurocirugía 2007; 18: 312-319.

Correspondencia postal: Juan F. Martínez-Lage. Servicio de Neurocirugía. Hospital Universitario "Virgen de la Arrixaca". 30120 El Palmar. Murcia. 